\title{
Evaluation of the early fluconazole treatment of candidemia protocol with automated short message service alerts: a before-and-after study
}

\author{
Jeong Rae Yoo ${ }^{1,2}$, Bo Ra Shin ${ }^{2}$, Sujin $\mathrm{Jo}^{2}$, and Sang Taek Heo ${ }^{1,2}$
}

${ }^{1}$ Department of Internal Medicine, Jeju National University School of Medicine, Jeju; ${ }^{2}$ Infection Control Unit, Jeju National University Hospital, Jeju, Korea
Received: August 7, 2019

Revised : September 23, 2019

Accepted: November 28, 2019

\section{Correspondence to}

Sang Taek Heo, M.D.

Department of Internal

Medicine, Jeju National

University School of Medicine,

15 Aran 13-gil, Jeju 63241, Korea

Tel: +82-64-754-8151

Fax: +82-64-717-1131

E-mail: neosangtaek@naver.com https://orcid.org/0000-0001-

8947-5069
Background/Aims: We introduced the Early Fluconazole Treatment in Candidemia (EFTC) protocol in August 2015 to improve the outcomes of patients with candidemia. This study evaluated the effectiveness of the EFTC protocol.

Methods: We conducted a retrospective before-and-after study among patients in the intensive care units and Hemato-Oncology and General Surgery wards of our hospital between January 2013 and December 2018. The EFTC protocol entailed sending an automatic notification by short message service, feedback to the responsible healthcare worker, and regular standardized education of medical staff. On receiving a notification, physicians prescribed empirical fluconazole immediately. The effectiveness of the EFTC protocol was evaluated by multivariate analysis of risk factors for 30-day mortality.

Results: Of 103 patients with candidemia, 50 were admitted before (pre-EFTC group) and 53 were admitted after (post-EFTC group) the introduction of the EFTC protocol. Patients' mean age \pm SD was $67.1 \pm 18.6$ years, and 55 (53.4\%) were male. The mean $\pm \mathrm{SD}$ time from Candida isolation to antifungal drug administration in the pre-EFTC and post-EFTC groups was $89.1 \pm 73.6$ and $-9.8 \pm 63.9 \mathrm{~min}-$ utes, respectively $(p=0.01)$. The 30 -day mortality in the pre-EFTC and post-EFTC groups was $54.5 \%$ (95\% confidence interval [CI], 42.3 to 66.7 ), and $37.6 \%$ (95\% CI, 26.1 to 49.1$)$, respectively $(p=0.05)$. In the multivariate analysis, implementation of the EFTC protocol was independently associated with a reduction in 30-day mortality (odds ratio, $0.27 ; 95 \% \mathrm{CI}$, 0.12 to $0.63 ; p=0.01$ ).

Conclusions: The early fluconazole treatment, monitoring, and education were effective in reducing mortality in patients with candidemia.

Keywords: Candidemia; Antifungal agents; Fluconazole

\section{INTRODUCTION}

Candidemia is associated with substantial morbidity and has a high mortality rate $[1,2]$. In hospitalized patients with candidemia, a delay in initiation of fluconazole therapy is associated with a high mortality rate [3]. In 2016, the Infectious Diseases Society of America
(IDSA) released updated guidelines on the management of Candida infections [2]. Currently, an echinocandin is recommended as initial therapy. The 2016 IDSA guidelines recommend testing all Candida isolates for azole susceptibility in patients with candidemia [2], Fluconazole is an acceptable alternative to an echinocandin as initial therapy in selected patients who are not critically 


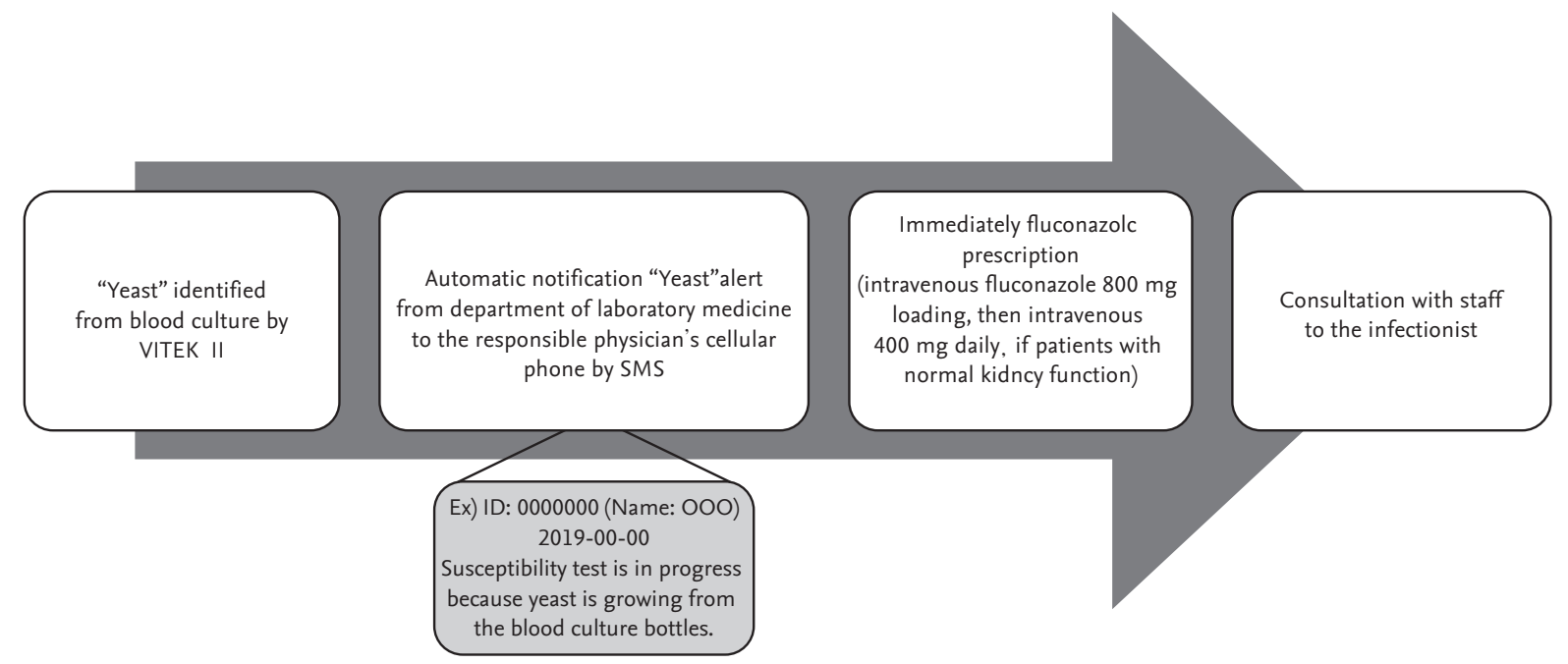

Figure 1. Protocol for early fluconazole treatment of patients with candidemia. SMS, short message service.

ill and who are considered unlikely to be infected by a fluconazole-resistant Candida species. Candida albicans, C. parapsilosis, and C. tropicalis are sensitive to fluconazole (> 90\% sensitivity) in the Asia-Pacific region [4]. On the basis of $100 \%$ susceptibility of candida isolates to fluconazole among patients with candidemia admitted to our hospital between 2013 and 2015, we decided to evaluate the Early Fluconazole Treatment in Candidemia (EFTC) protocol among patients with confirmed candidemia.

\section{METHODS}

\section{Study design and patient population}

We conducted a retrospective before-and-after study to evaluate the effects of the implementation of the EFTC protocol in the medical and surgical intensive care unit (ICU) and the departments of Hemato-Oncology and General Surgery in a tertiary center in Jeju Province in South Korea between January 2013 and December 2018. The study protocol was approved by the Institutional Review Board of Jeju National University Hospital (JNUH 17-12-OO1). Informed consent was waived because the study was based on a retrospective review of clinical and laboratory records.

To be eligible for inclusion in the study, patients had to have been $>18$ years and to have been admitted to the hospital with candidemia. We excluded patients with other fungus and Candida species from other specimen such as sputum, urine, and wound. In addition, patients admitted from August 2015 onward had to have been treated according to the EFTC protocol.

\section{Early fluconazole treatment in candidemia protocol}

The EFTC protocol included an automatic notification "Blood culture: yeast" by short message service (SMS) from the Department of Laboratory Medicine to the responsible physician's cellular phone; standardized education about the importance for early antifungal treatment of candidemia by an infection control specialist every 6 months; and feedback to the relevant healthcare workers, such as doctors and nurses, in the departments of Hemato-Oncology and General Surgery, and the ICUs about antifungal coverage rate, adequate empirical antifungal drug, time from blood culture to antifungal drug administration, and time from yeast alert to antifungal drug administration. On receiving the SMS notification, the physician immediately prescribed an 800 mg loading dose of intravenous fluconazole, followed by intravenous $400 \mathrm{mg}$ daily (in patients with normal renal function), followed by consultation with an infection control specialist (Fig. 1).

\section{Variables and data collection}

Blood cultures were performed using the Bact/Alert $3 \mathrm{D}$ system (bioMérieux Inc., Marcy l'Étiole, France), and the cultures were incubated for 24 to 48 hours and then inoculated onto blood agar. Colonies were collected from 
the plates for identification using an automated system (VITEK II, bioMérieux). Clinical data from the patients were obtained using a structured case report form, which included demographics, underlying diseases, diagnosis, results of laboratory tests, drug susceptibility tests of Candida species, antifungal drug treatment, duration of hospitalization, Multiple Organ Dysfunction Score (MODS), Charlson comorbidity index (CCI), Candida score, time to initiation of fluconazole after the notification alert had been sent to physicians (in the post-EFTC group), and 30-day mortality.

\section{Definitions}

A calculation of the antifungal coverage rate was included as the antifungal drugs of fluconazole, echinocandin, amphotericin B deoxycholate, and lipid formulations of amphotericin B in the pre-EFTC group. In the postEFTC group, it included the antifungal drug of fluconazole. Adequate empirical fluconazole therapy for candidemia was defined as initiation of fluconazole therapy after identification of candidemia, at a dose $\geq 400 \mathrm{mg} /$ day (in individuals with normal renal function), and isolation of an organism from the Candida genus that was determined to be sensitive to fluconazole on the basis of an in vitro susceptibility test. Time from blood culture to antifungal drug administration was defined as the number of days from the culture of the first blood sample to the administration of antifungal drugs. Time from Candida isolation to antifungal drug administration was defined as the number of days from the culture of the first blood sample positive for Candida species to the administration of antifungal drugs. Time from onset of yeast to antifungal drug administration was defined as the number of days from the culture of the first blood sample positive for yeast to the initiation of fluconazole. Empirical antifungal therapy was defined as any antifungal therapy started before the identification of the Candida species. Duration of antifungal drug was defined as the number of days from the initiation of empirical antifungal drugs to the end of definite antifungal drug administration. The Candida score is a scoring system that clinicians use to differentiate between patients with Candida colonization and those with ongoing but occult invasive fungal infection when considering starting early fungal treatment [5].

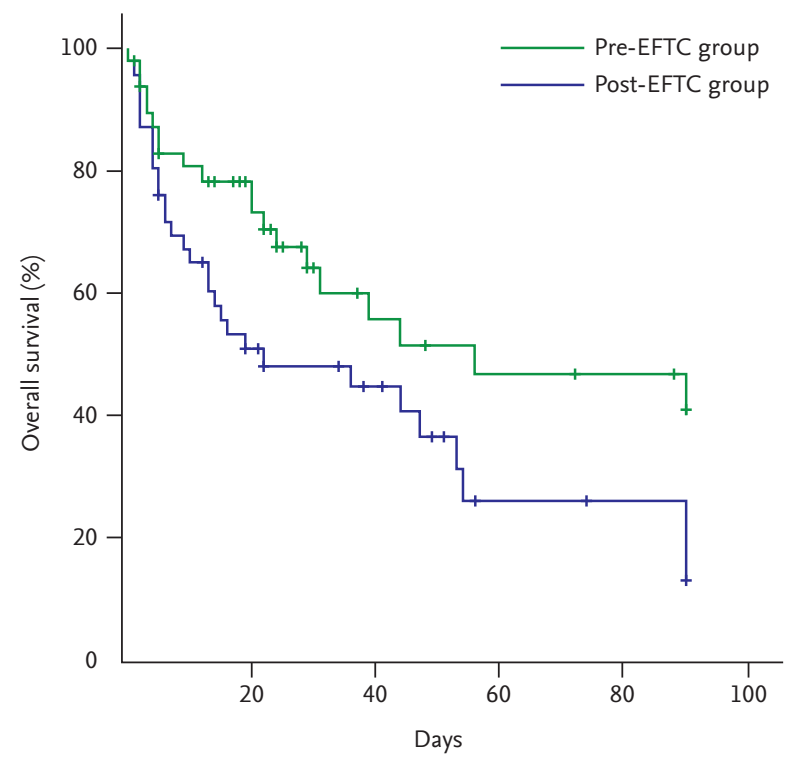

Figure 2. Kaplan-Meier plots comparing the survival in the pre-Early Fluconazole Treatment of Candidemia (EFTC) and post-EFTC groups of patients with candidemia. The 30-day mortality in the pre-EFTC and post-EFTC groups was $54.5 \%$ (95\% confidence interval [CI], 42.3 to 66.7 ), and $37.6 \%$ (95\% CI, 26.1 to 49.1$)$, respectively $(p=0.05)$.

\section{Statistical analysis}

Data were analyzed using SPSS version 20.0 (IBM Corp., Armonk, NY, USA). Outcome measures were compared using either a chi-square or a $t$ test for categorical and continuous measures, respectively. Multiple logistic regression analysis of Candida risk factors and other variables associated with mortality $(p<0.05)$ in the univariate analyses was performed to identify independent predictors of 30-day mortality. The Kaplan-Meier method was used to calculate 30-day survival probability in both groups. A $p$ value $<0.05$ was considered to be statistically significant.

\section{RESULTS}

\section{Baseline characteristics in pre- and post-EFTC group}

A total of 109 patients with candidemia were admitted to our hospital during the study period. Fifty patients admitted before August 2015 were included in the preEFTC group. Of the 59 patients admitted from August 2015, 53 were included in the post-EFTC group and six were excluded because they were lost to follow-up. Fi- 
Table 1. Comparison of baseline characteristics and clinical outcomes in the pre- and post-EFTC groups

\begin{tabular}{|c|c|c|c|}
\hline Characteristic & $\begin{array}{l}\text { Pre-EFTC group } \\
(\mathrm{n}=50)\end{array}$ & $\begin{array}{l}\text { Post-EFTC group } \\
\qquad(\mathrm{n}=53)\end{array}$ & $p$ value \\
\hline Age, yr & $70.85 \pm 11.58$ & $64.1 \pm 17.3$ & 0.07 \\
\hline Male sex & $25(54 \cdot 3)$ & $29(53.7)$ & 0.95 \\
\hline Charlson comorbidity index & $6.5 \pm 3 \cdot 3$ & $5 \cdot 0 \pm 3 \cdot 3$ & 0.02 \\
\hline \multicolumn{4}{|l|}{ Risk factors of candidemia } \\
\hline Total parenteral nutrition & $22(47.8)$ & $23(45 \cdot 1)$ & 0.79 \\
\hline Abdominal surgery & $14(30.4)$ & $5(9.8)$ & $<0.001$ \\
\hline Previous Candida infection & $3(6.5)$ & $4(7.8)$ & 0.80 \\
\hline Central venous catheter & $36(78.3)$ & $35(68.6)$ & 0.28 \\
\hline Chemotherapy & $9(19.6)$ & $13(25 \cdot 5)$ & 0.49 \\
\hline Steroid use & $5(10.9)$ & $17(33.4)$ & 0.01 \\
\hline Candida risk score & $1.5 \pm 1.4$ & $1.2 \pm 1.2$ & 0.23 \\
\hline Candida species & & & 0.82 \\
\hline C. albicans & $20(43.5)$ & $22(40.7)$ & \\
\hline C. parapsilosis & $8(17 \cdot 4)$ & $9(16.7)$ & \\
\hline C. krusei & $1(2.2)$ & $2(3.7)$ & \\
\hline C. glabrata & $7(15.2)$ & $5(18.5)$ & \\
\hline C. tropicalis & $7(15.2)$ & $7(13.0)$ & \\
\hline Others & $3(6.5)$ & $8(14.8)$ & \\
\hline Fluconazole sensitive & $46(100)$ & $44(91.7)^{\mathrm{a}}$ & 0.87 \\
\hline \multicolumn{4}{|l|}{ Outcomes } \\
\hline Antifungal coverage rate & $34(73 \cdot 9)$ & $53(100)$ & 0.01 \\
\hline Adequate empirical antifungal drug & $33(71.7)$ & $47(98)^{\mathrm{a}}$ & 0.01 \\
\hline Time from blood culture to antifungal drug administration, hr & $107.6 \pm 125.1$ & $87.4 \pm 68.8$ & 0.41 \\
\hline Time from Candida isolation to antifungal drug administration, hr & $89.1 \pm 73.6$ & $-9.8 \pm 63.9^{b}$ & 0.01 \\
\hline Duration of antifungal drug & $11.5 \pm 5.7$ & $15 \cdot 7 \pm 6.4$ & 0.05 \\
\hline MODS & $5 \cdot 5 \pm 4.1$ & $3.4 \pm 3.6$ & 0.01 \\
\hline ICU admission & $20(43.4)$ & $26(48.1)$ & 0.07 \\
\hline Mechanical ventilation & $15(32.6)$ & $16(29.6)$ & 0.17 \\
\hline Treated with vasopressors & $21(45 \cdot 7)$ & $13(24.1)$ & 0.02 \\
\hline Hospital days & $44.2 \pm 30.4$ & $69.6 \pm 104.8$ & 0.11 \\
\hline 30-day mortality, \% & $54.5(42.3-66.7)$ & $37.6(26.1-49.1)$ & 0.02 \\
\hline
\end{tabular}

Values are presented as mean $\pm \mathrm{SD}$, number $(\%)$, or mean ( $95 \%$ confidence interval).

EFTC, Early Fluconazole Treatment in Candidemia; MODS, Multiple Organ Dysfunction Score; ICU, intensive care unit.

${ }^{a}$ Forty-four Candida species were susceptible to fluconazole among 48 patients with available susceptibility results.

${ }^{\mathrm{b}}$ In the post-EFTC group, fluconazole was administrated before Candida isolation.

nally, a total of 103 patients (50 before and 53 after) were included in the analysis. The characteristics of the two groups are summarized in Table 1 . There was no statistically significant difference between the two groups in demographic characteristics, risk factors of candidemia, department of admission, or their laboratory results on admission. However, patients in the post-EFTC group had a significantly lower CCI and MODS than those in the pre-EFTC group. The distribution by hospital department (General Surgery or Hemato-Oncology); distribution of Candida species; and the proportion of patients who had a central catheter removed did not differ significantly between groups. The mean starting dose of fluconazole (excluding the loading dose) also did not differ significantly between groups. 
Table 2. Multivariate analysis of risk factors associated with 30-day mortality in patients with candidemia

\begin{tabular}{|c|c|c|c|c|c|c|}
\hline \multirow{2}{*}{ Variable } & \multicolumn{3}{|c|}{ Model I } & \multicolumn{3}{|c|}{ Model II ${ }^{\mathrm{b}}$} \\
\hline & OR & $95 \% \mathrm{CI}$ & $p$ value & OR & $95 \% \mathrm{CI}$ & $p$ value \\
\hline Charlson comorbidity index $\geq 6$ & 2.90 & $1.24-6.77$ & 0.01 & 2.94 & $1.20-7.16$ & 0.02 \\
\hline MODS $\geq 6$ & 2.17 & $0.93-5.06$ & 0.07 & 2.43 & $0.88-6.66$ & 0.08 \\
\hline Candida risk score $\geq 3$ & 1.06 & $0.34-3 \cdot 32$ & 0.92 & 1.02 & $0.35^{-2.96}$ & 0.97 \\
\hline Time to fluconazole initiation $\geq-5 \mathrm{hr}^{\mathrm{a}}$ & 3.04 & $1.11-8.35$ & 0.03 & 4.45 & $1.78-7.87$ & 0.02 \\
\hline ICU admission & 1.12 & $0.47-2.62$ & 0.80 & 1.07 & $0.21-2.54$ & 0.88 \\
\hline Mechanical ventilation & 1.07 & $0.45-2.54$ & 0.88 & 1.11 & $0.34-2.78$ & 0.82 \\
\hline Vasopressor & 2.59 & $1.10-6.10$ & 0.03 & 2.43 & $1.16-6.32$ & 0.05 \\
\hline Implementation of EFTC protocol & 0.27 & $0.12-0.63$ & 0.01 & & & \\
\hline
\end{tabular}

OR, odds ratio; CI, confidence interval; MODS, Multiple Organ Dysfunction Score; ICU, intensive care unit; EFTC, Early Fluconazole Treatment of Candidemia.

Time from Candida isolation on blood culture to fluconazole initiation.

${ }^{\mathrm{b}}$ Implementation of the EFTC protocol was excluded in multivariate analysis.

\section{Effectiveness of the EFTC protocol}

The antifungal coverage rate; and the use adequate empirical antifungal drug was significantly higher; while the time from Candida isolation to antifungal drug initiation was significantly shorter (Table 1), and 30-day mortality rate was significantly lower in the post-EFTC group than the pre-EFTC group (Fig. 2). However, the mean time from blood culture to antifungal drug initiation did not differ significantly by group.

\section{Risk factors for 30-day mortality}

Univariate analysis of risk factors showed that age (odds ratio [OR], 0.21; 95\% confidence interval [CI], 0.99 to 1.04; $p=0.77$ ), total parenteral nutrition (OR, 1.33; 95\% CI, 0.59 to $2.97 ; p=0.49$ ), abdominal surgery (OR, o.84; 95\% CI, 0.33 to $2.16 ; p=0.72$ ), previous Candida infection (OR, $0.61 ; 95 \% \mathrm{CI}$, 0.11 to $3.29 ; p=0.61)$, central catheter insertion (OR, 0.83 ; $95 \% \mathrm{CI}, 0.33$ to $2.06 ; p=0.68$ ), chemotherapy (OR, 1.10; $95 \%$ CI, 0.42 to $2.91 ; p=0.84$ ), ICU admission (OR, 1.78; 95\% CI, 0.77 to 3.89; $p=0.19$ ), and Candida score $\geq 3$ (OR, 1.56; 95\% CI, 0.57 to $3.78 ; p=0.20$ ) were not significantly associated with the 30 -day mortality. CCI $\geq 6$ points (OR, 3.24; 95\% CI, 1.26 to 7.54; $p=0.01), \mathrm{MODS} \geq 6$ points (OR, 3.04; 95\% CI, 1.18 to 7.11; $p=0.01$ ), and time to fluconazole initiation $\geq-5$ hours (OR, 4.89; $95 \% \mathrm{CI}$, 1.45 to $9.57 ; p=0.01$ ) were associated with a significantly higher 30 -day mortality.

The results of the multivariate logistic regression analysis to identify factors independently associated with 30-day mortality are shown in Table 2. When multivariate analysis was performed to compare the relative contribution of risk factors associated with 30-day mortality Implementation of EFTC protocol was independently associated with a significantly lower 30-day mortality (OR, 0.27; 95\% CI, 0.12 to $0.63 ; p=0.01$ ). An antifungal administration after Candida isolation from blood culture, use of vasopressor, and a CCI $\geq 6$ points was independently associated with a significantly higher 30-day mortality.

\section{DISCUSSION}

Previous studies have shown that sepsis, multiple organ failure, Candida colonization, abdominal surgery, inadequate empirical antifungal treatment, and untimely treatment are independent risk factors for candidemia $[6,7]$. The 2016 IDSA guidelines for the management of candidiasis recommend that patients with candidemia in ICUs be given an echinocandin as empiric treatment [2]. A study addressing the effects of delayed treatment found that a delay of $>12$ hours was an independent predictor of in-hospital mortality [7]. Fluconazole is an acceptable alternative for patients who have had no recent azole exposure and are not colonized with azole-resistant Candida species. On the basis of 100\% fluconazole sensitivity in patients with candidemia treated at our hospital between January 2013 and July 2015, we chose 
fluconazole as the initial empirical regimen for treating candidemia in our patients. However, the recent emergence of multidrug-resistant Candida species will complicate the choice of antifungal therapy $[3,8]$. In addition, physicians in South Korea are reluctant to use antifungal agents without confirmation of infection because of the toxicity of amphotericin B and the high cost of the new echinocandins, which is generally not covered by health insurance. Encouragingly, there was a significant improvement in the early administration of antifungal drugs, 30-day mortality, antifungal coverage, and duration of antifungal drug administration following the introduction of the EFTC protocol but the time to initiation of fluconazole after a positive blood culture did not change significantly.

As earlier antifungal therapy is associated with better overall outcomes in patients with candidemia, we introduced a regular education and feedback to staff in the relevant departments. There was a substantial reduction in the time from identification of Candida in the blood culture to initiation of fluconazole after implementation of the EFTC protocol. However, there was significantly higher of comorbidity and disease severity with multiple organ dysfunction in the pre-EFTC group, and antifungal drug was not administered in $26.1 \%$ of patients, of whom half died before the final report of the blood culture was released. Even if above differences of baseline characteristics between both groups, we accomplished a full coverage of antifungal drug administration in patients with candidemia, adequate empirical antifungal drug, and adequate duration of antifungal drug in the post-EFTC group. In addition, the infection control team will continue to conduct continuous monitoring to further reduce the time to fluconazole initiation. The results show that implementation of the EFTC protocol led to a considerable reduction of 30-day mortality in patients with candidemia.

This study has a few limitations. First, it included a relatively small number of patients. Second, it did not have data on the time of onset of yeast report in preEFTC group, so we were unable to compare the time from the onset of candidemia to fluconazole initiation in the two groups. Third, we could not evaluate the difference between groups in the severity of candidemia, or the effectiveness of antifungal therapy due to the retrospective study design. Fourth, we did not compare central catheter-related infection caused by Candida species. Finally, we were unable to estimate the indirect effects on the enforcement of healthcare-associated infection control and prevention related to factors such as improvement of hand hygiene, hospital environment cleaning, disposal of medical devices, and change of disinfectants during the post-EFTC period. A prospective, multicenter study with a large sample size is needed to further evaluate the effectiveness of the EFTC protocol.

In conclusion, early antifungal administration, improvement of antifungal coverage, adequate duration of antifungal drug administration, and educating physicians on the use of antifungal therapy for candidemia was associated with a good outcome in patients with candidemia. Even with limited resources for infection control, this protocol can readily be applied in clinical practice.

\section{KEY MESSAGE}

1. Implementation of a protocol to promote early administration of fluconazole in hospitalized patients with candidemia was associated with a reduction in 30-day mortality.

2. Implementation of the protocol was also associated with a reduction in the time to starting fluconazole after identifying Candida on blood culture, and an improvement in fluconazole coverage.

3. The protocol can readily be applied in clinical practice, even in limited-resource settlings.

\section{Conflict of interest}

No potential conflict of interest relevant to this article was reported.

\section{Acknowledgments}

This work was supported by a research grant from the Jeju National University Hospital in 2017.

\section{REFERENCES}

1. Yang ZT, Wu L, Liu XY, et al. Epidemiology, species distribution and outcome of nosocomial Candida spp. blood- 
stream infection in Shanghai. BMC Infect Dis 2014;14:241.

2. Pappas PG, Kauffman CA, Andes DR, et al. Clinical practice guideline for the management of candidiasis: 2016 update by the Infectious Diseases Society of America. Clin Infect Dis 2016;62:e1-e5o.

3. Garey KW, Rege M, Pai MP, et al. Time to initiation of fluconazole therapy impacts mortality in patients with candidemia: a multi-institutional study. Clin Infect Dis 2006;43:25-31.

4. Wang H, Xu YC, Hsueh PR. Epidemiology of candidemia and antifungal susceptibility in invasive Candida species in the Asia-Pacific region. Future Microbiol 2016;11:14611477 .

5. Leon C, Ruiz-Santana S, Saavedra P, et al. A bedside scor- ing system ("Candida score") for early antifungal treatment in nonneutropenic critically ill patients with Candida colonization. Crit Care Med 2006;34:730-737.

6. Chae YT, Jeong SJ, Ku NS, et al. Risk factors and prognosis for persistent candidemia without catheter colonization. Infect Chemother 2011;43:349-354.

7. Morrell M, Fraser VJ, Kollef MH. Delaying the empiric treatment of candida bloodstream infection until positive blood culture results are obtained: a potential risk factor for hospital mortality. Antimicrob Agents Chemother 2005;49:3640-3645.

8. Ostrosky-Zeichner L, Kullberg BJ, Bow EJ, et al. Early treatment of candidemia in adults: a review. Med Mycol 2011;49:113-120. 